\title{
Empleo de la estrategia puzle de Aronson para fomentar la cooperación en grupos
}

\section{Francesc A. Esteve-Turrillas, Sergio Armenta}

Departamento de Química Analítica, Universitat de València, Edificio Jeroni Muñoz, c/ Dr. Moliner 50, 46100 Burjassot, España (francesc.a.esteve@uv.es, sergio.armenta@uv.es)

\begin{abstract}
The main objective of the present communication was the implementation of an strategy based on the Puzzle of Aronson in the tutorials corresponding to the Food Additives course of the Degree in Science and Food Technology. The competences of students regarding cooperative learning increased using this dynamic and functional methodology. This type of methodologies are easy to apply in heterogeneous groups of students and are easy to adapt to the characteristics of the classroom.
\end{abstract}

Keywords: Cooperative learning, Aronson Puzzle, Group work, Food additives

\begin{abstract}
Resumen
Se ha implementado una estrategia tipo Puzle de Aronson en las tutorías correspondientes a la asignatura Aditivos Alimentarios del Grado de Ciencia y Tecnología de Alimentos. Con esta metodología dinámica y funcional se han aumentado las competencias del alumnado referentes al trabajo cooperativo, desarrollo de habilidades sociales y de comunicación, y resolución de conflictos. Este tipo de metodologías resultan fáciles de aplicar en grupos heterogéneos de alumnos y resultan fáciles de adaptar a las características del aula.
\end{abstract}

Palabras clave: Aprendizaje cooperativo, Puzle de Aronson, Trabajo en grupo, Aditivos Alimentarios

\section{Introducción}

El aprendizaje cooperativo es un método de aprendizaje basado en el trabajo en equipo en el que los alumnos trabajan conjuntamente para lograr determinados objetivos comunes de los que son responsables todos los miembros del grupo. El aprendizaje cooperativo se basa en la teoría constructivista en la que se otorga un papel fundamental al alumno, que es actor principal de su propio proceso de aprendizaje (Kagan, 1994). El trabajo cooperativo lleva implícito la interrelación entre los estudiantes de un grupo para trabajar juntos y lograr objetivos compartidos, por lo que los resultados obtenidos resultan beneficiosos, tanto para cada miembro, como para la totalidad del grupo (Johnson, 1991). La técnica del puzle de Aronson emplea una metodología dinámica y funcional que permite aumentar las competencias del alumnado referentes al trabajo cooperativo, en el que se confrontan diversos puntos de vista y se fomentan situaciones de conflicto y discusión. En esta estrategia de aprendizaje los estudiantes trabajan en pequeños grupos, preferiblemente 
no superiores a 4 personas. Se realiza un reparto cooperativo de tareas, lo que fomenta la responsabilidad de cada miembro, las relaciones sociales y la solución de conflictos internos. La técnica del Puzle de Aronson consta de tres etapas claramente diferenciadas (García, 2001). En la primera de ellas se divide a los alumnos en grupos aleatoriamente para solucionar un problema. En una segunda etapa, cada uno de los miembros del grupo se separa en diferentes grupos de expertos para que aprendan sobre una temática concreta, debatan las posibles dudas y recopilen información complementaria. En la tercera etapa, los miembros del grupo de trabajo inicial se reúnen, con conocimientos especializados cada uno de ellos, y se disponen a la resolución del problema de tipo práctico. De esta forma todos los estudiantes se reubican en grupos integrados por un experto de cada una de las áreas temáticas a considerar $\mathrm{y}$ han de ser capaces de transmitir efectivamente sus conocimientos al resto de compañeros para resolver el problema propuesto. En esta etapa es crucial que todos los miembros del grupo de trabajo discutan las posibles soluciones del problema, se generen y resuelvan conflictos, y se llegue a un resultado final consensuado por todos los miembros. El conflicto es empleado como situación de aprendizaje, ya que fomenta las relaciones humanas y la asertividad. La superación de un conflicto consolida al grupo, lo prepara y anima para enfrentarse en un fututo a problemas más complejos.

Esta estrategia de aprendizaje debe ser evaluada de forma independiente, por una parte el proceso de aprendizaje cooperativo, y por otra la capacidad de resolución del problema propuesto. La evaluación de la productividad del grupo, obliga a que los alumnos dependan unos de otros para lograr su objetivo y de esta forma se reduce la competencia interna entre sus integrantes. Los objetivos que se persiguen con el empleo de una estrategia tipo puzle de Aronson para el trabajo en grupo de estudiantes se basan en (Traver, 2004):

- Mejorar el aprendizaje cooperativo.

- Desarrollar habilidades sociales, asertivas y de comunicación.

- Desarrollar la solidaridad y el compromiso cívico entre el alumnado.

- Fomentar la autonomía en el aprendizaje.

- Aumentar el rendimiento académico.

- Desarrollar competencias académicas y profesionales.

\section{Objetivos}

El objetivo de la presente comunicación consiste en la implementación de una estrategia tipo Puzzle de Aronson en las tutorías correspondientes a la asignatura Aditivos Alimentarios del Grado de Ciencia y Tecnología de los Alimentos de la Universitat de València. Con esta metodología, dinámica $\mathrm{y}$ funcional, se pretende aumentar las competencias del alumnado referentes a aprendizaje cooperativo, desarrollar habilidades sociales y de comunicación, fomentar la autonomía en el aprendizaje, y también aumentar el rendimiento académico.

(c)) BY-NC-ND 2017, Universitat Politècnica de València 


\section{Desarrollo de la innovación}

\subsection{Contexto de aprendizaje}

La propuesta de trabajo cooperativo, basada en el empleo de estrategias tipo Puzle de Aronson, se ha aplicado a la asignatura Aditivos Alimentarios correspondiente al Grado de Ciencia y Tecnología de los Alimentos que se imparte en la Facultad de Farmacia de la Universitat de València. La asignatura incluye 25 horas de teoría, 15 horas de prácticas de laboratorio, 2 horas de seminario coordinado y 2 horas de tutoría presencial. La asignatura es optativa y se imparte en el cuarto curso del grado con una frecuencia de 2-3 horas semanales. El número habitual de alumnos es de aproximadamente 25-30 miembros, siendo 29 en el curso 2016-2017. La asignatura se imparte en una aula con asientos y mesas fijos con una capacidad de 120 personas. El perfil de los estudiante en el curso 2016-2017 es el siguiente: $68.97 \%$ mujeres y $31.03 \%$ hombres, edad promedio de 23.34 años, un $93.10 \%$ no realiza ningún tipo trabajo renumerado, y un $13.79 \%$ proceden de programas internacionales de intercambio (Erasmus,...).

\subsection{Contenidos de la asignatura}

Los contenidos que se imparten en la asignatura Aditivos Alimentarios se dividen en 13 temas, correspondientes a las distintas categorías de aditivos alimentarios permitidos en la Unión Europea, así como un tema inicial introductorio y dos finales relativos a evaluación toxicológica y legislación actual. El título de cada tema con el número de horas aproximado se muestra en la Tabla 1.

Tabla 1. Temas correspondientes a la asignatura Aditivos Alimentarios

\begin{tabular}{lll}
\hline Tema & Título & Horas \\
\hline Tema 1 & Introducción & 1 \\
Tema 2 & Aditivos antioxidantes & 3 \\
Tema 3 & Aditivos antimicrobianos. & 3 \\
Tema 4 & Agentes depresores de la actividad de agua. & 1 \\
Tema 5 & Antiendurecedores. & 2 \\
Tema 6 & Aromatizantes y potenciadores del sabor. & 2 \\
Tema 7 & Edulcorantes. & 2 \\
Tema 8 & Colorantes & 2 \\
Tema 9 & Espesantes y gelificantes & 2 \\
Tema 10 & Emulgentes & 1 \\
Tema 11 & Auxiliares tecnológicos de fabricación & 2 \\
Tema 12 & Evaluación toxicológica & 2 \\
Tema 13 & Legislación relativa a aditivos & 2 \\
\hline
\end{tabular}

(c) $)$ EY-NC-ND 2017, Universitat Politècnica de València 


\subsection{Temporalización de la actividad}

La actividad propuesta se ha realizado en dos sesiones de tutorías presenciales en el aula (1 hora cada sesión) que se realizan a mitad del semestre en el que aproximadamente ya se ha impartido la mitad del temario. Las dos tutorías han tenido lugar en un plazo corto de tiempo (1-2 semanas), por lo que los alumnos han tenido un plazo de tiempo adecuado para la asimilación de los conceptos adquiridos y meditación de las ideas concebidas. Asimismo, ha permitido la búsqueda fuera del aula de conocimientos adicionales y complementarios al temario impartido en la asignatura para su aplicación en la segunda tutoría.

\subsection{Descripción de la tarea}

En las tutorías de esta asignatura se ha desarrollado una actividad basada en el puzle de Aronson para fomentar el trabajo cooperativo. Los alumnos se dividen en dos grupos de 15 personas aproximadamente para realizar las tutorías, por lo que el número de alumnos fue reducido. La actividad propuesta consiste en la resolución por grupos de diferentes problemas prácticos relacionados con el empleo de Aditivos Alimentarios en la formulación de alimentos.

La idea general consiste, en una primera etapa, en la selección aleatoria de 4 personas para formar un grupo al que se le asignará la tarea de resolver un problema de tipo práctico. En una segunda etapa cada uno de los miembros del grupo inicial se divide en 4 grupos de expertos para que se formen en una materia específica (ver esquema en Figura 1). En este caso cada grupo se centró en una categoría de aditivos alimentarios, como antioxidantes, antimicrobianos, edulcorantes, y colorantes. Cada grupo se sitúa en los extremos del aula para no interferir entre ellos y el profesor va coordinando las actividades dentro de cada grupo de expertos, para orientar al grupo y dirigir su aprendizaje en aquellos temas clave. Durante esta etapa, la formación consiste en la consulta y discusión de los apuntes de clase, clasificando los aditivos por naturaleza, riesgo, frecuencia de uso, características físico-químicas y coste. Tras la primera sesión de tutorías, los expertos en aditivos buscan información adicional fuera del aula para complementar los conocimientos necesarios para poder ser empleados en la segunda etapa. Esta búsqueda de información se realiza tanto por bibliografía reglada (libros, revistas, periódicos), como no reglada (consulta en internet, foros, redes sociales, etc).

En una tercera fase ( $2^{\circ}$ día de tutoría) cada uno de los expertos formados en cada tipo de aditivos alimentarios se reagrupa en los cuatro grupos de trabajo iniciales que abordarán la resolución de un problema relacionado con la formulación de los aditivos adecuados para diferentes tipos de alimentos: productos cárnicos, vegetales, panadería y bollería, y lácteos (ver Figura 1). En esta etapa, los alumnos disponen de diversas etiquetas reales de alimentos correspondientes a dichas categorías y proceden a una evaluación del tipo de aditivos que lleva, el por qué, posibles riesgos toxicológicos, consecuencias de su retirada de la formulación, y propuesta de posibles sustitutos. La Figura 2 muestra alguna de las etiquetas modelo empleadas en la actividad propuesta. Adicionalmente, existen

(c)) BY-NC-ND 2017, Universitat Politècnica de València 
efectos sinérgicos en el uso de aditivos de distintas familias, por lo que se fomenta la discusión para ver qué aditivos son más necesarios que otros y cuáles de ellos se complementan. Finalmente, se les propuso un caso de un alimento ficticio en el que cada grupo de alumnos debía de proponer de forma consensuada y razonada la adición de aditivos si fuera necesario y el por qué.

\section{Grupo de expertos: 1a sesión}

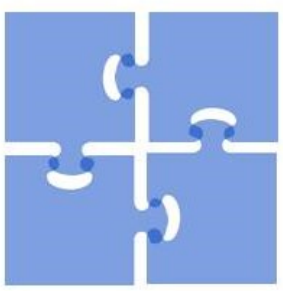

Antioxidantes

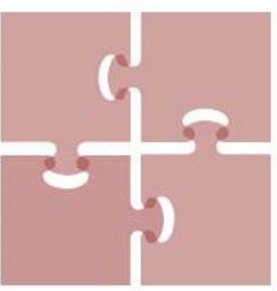

Antimicrobianos

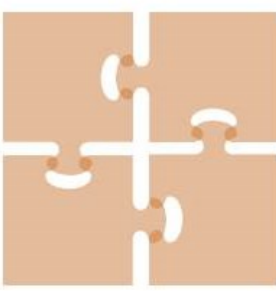

Edulcorantes

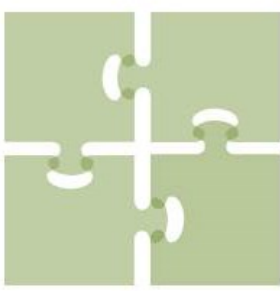

Colorantes

\section{Grupo de trabajo: 2a sesión}

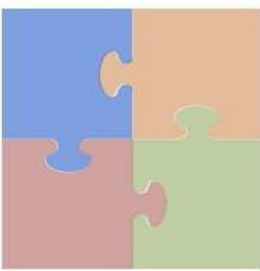

Productos cárnicos

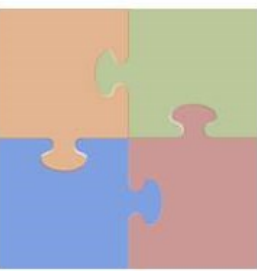

Vegetales

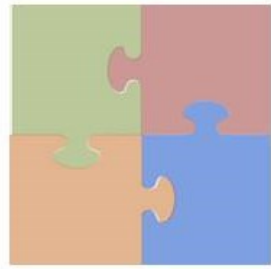

Panadería y bollería

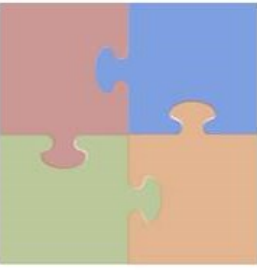

Lácteos

Fig. 1 Esquema de los grupos de expertos y de trabajo seleccionados en la actividad propuesta.

\section{Resultados}

\subsection{Resultados de aprendizaje}

La presente tarea pretende en su primera sesión afianzar los conceptos teóricos que se imparten en la asignatura a través de la autoformación de expertos en aquellos aditivos alimentarios más frecuentes e importantes, que se imparten en los primeros temas del curso (Temas 2: Antioxidantes, Tema 3: Antimicrobianos, Tema 7: Edulcorantes, y Tema 8: Colorantes). Considerando la temática elegida en la actividad, existe gran cantidad de información no reglada, sobre todo en búsquedas por internet y foros de opinión que permitió al grupo de expertos discutir la veracidad o falsedad parcial de los datos encontrados en la red. Esto contribuyó a incrementar el interés del estudiante por la búsqueda e interpretación de mayor cantidad de información, lo que fomenta su capacidad crítica, que se complementó con los conocimientos impartidos en las clases teóricas. Además, el empleo de estas metodologías de aprendizaje fomenta el estudio continuado de la materia, de forma que el alumnado no solo memoriza conceptos, sino que madura el conocimiento y fomenta su aplicación a casos reales. 


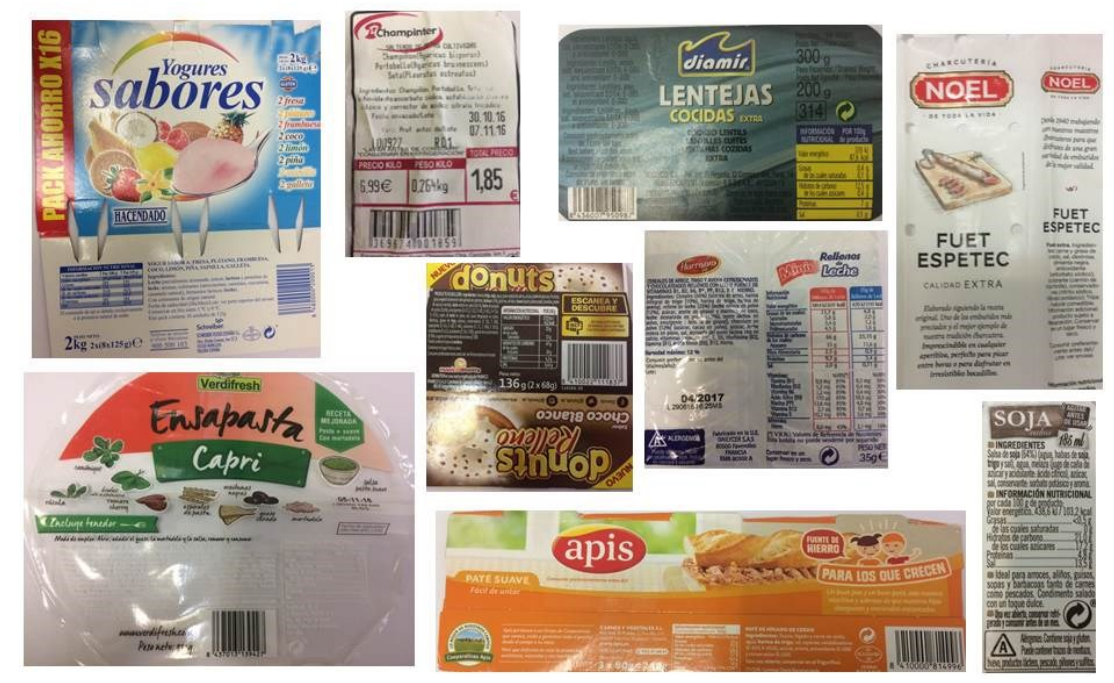

Fig. 2. Ejemplo de alguna de las etiquetas empleadas.

En la segunda sesión se aplicaron los conocimientos adquiridos por los distintos expertos en aditivos a etiquetas reales de alimentos que se les proporcionó en el aula. Los grupos de trabajo, discutieron la adecuación de los aditivos alimentarios presentes en cada producto y valoraron la posible eliminación/sustitución de los aditivos presentes. Finalmente, se les propuso un alimento modelo a cada grupo de trabajo para que decidieran por consenso y de una forma razonada los aditivos que debía incluir en su formulación para conseguir una elevada vida útil del alimento manteniendo unas características organolépticas adecuadas. Esto permitió que cada uno de los expertos defendiera y justificara la presencia o no de determinados aditivos alimentarios críticos en la formulación del alimento, adquiriendo competencias que les resultarán de gran utilidad en el desarrollo de su futura carrera profesional.

Durante la implantación de la tarea propuesta, sobre todo en la primera sesión de turorías, a los alumnos le costó un poco entender el proceso que se les proponía, sin embargo durante la segunda sesión se observó una dinámica mucho mas fluída. Por lo que para el año que viene, se emplearán unos minutos en las clase de teoría para explicarles el el fundamento del trabajo cooperativo propuesto.

A través del empleo de la técnica de trabajo cooperativo propuesta se desarrollaron competencias y habilidades sociales que figuran en la correspondiente guía docente de la asignatura, tales como:

- Capacidad de aplicar los conocimientos a la práctica.

- Razonamiento crítico que les permita emitir juicios argumentados y defenderlos con rigor y tolerancia.

- Capacidad de trabajar en grupo, responsabilidad compartida, gestión de conflictos, y propuesta de soluciones objetivas.

(c)) BY-NC-ND 2017, Universitat Politècnica de València 


\subsection{Evaluación de la actividad}

El aprendizaje por trabajo cooperativo se ha incorporado a la asignatura de Alimentos Alimentarios por primera vez en el curso 2016-2017 dentro de las 2 horas correspondientes a tutorías. Durante este primer año de aplicación de la actividad la evaluación de los resultados de aprendizaje obtenidos ha sido meramente cualitativa. Respecto al rendimiento académico, los resultados han estado en sintonía con cursos anteriores, con $3.4 \%$ matrícula de honor, $10.3 \%$ sobresaliente, $68.9 \%$ notable, 10.3 aprobado, y $6.9 \%$ no presentado. Gracias a la actividad propuesta se ha observado un aumento en el interés de los alumnos por incrementar sus conocimientos teóricos relativos al empleo de aditivos alimentarios y justificar su presencia en los alimentos. Fomentando su estudio en actividades del día a día tales como realizar la compra o seleccionar el alimento más saludable para la dieta. Debido a la respuesta favorable y elevado interés mostrado por los alumnos se pretende incorporar esta actividad al desarrollo de la asignatura en cursos venideros y evaluarla de una forma cuantitativa.

Considerando los resultados de aprendizaje que se trabajan con la actividad propuesta se propone una evaluación de tipo mixta con una parte formativa para valorar solo el proceso de aprendizaje (trabajo cooperativo), y otra sumativa para valorar la viabilidad y adecuación de las propuestas finales desarrolladas por cada grupo de trabajo. Además de la evaluación del profesor, la autoevaluación del alumno resulta clave para conocer lo útil que ha sido esta herramienta para fomentar el trabajo cooperativo. Con el objeto de cuantificar la evolución de las habilidades y competencias adquiridas por los alumnos respecto a su capacidad para realizar trabajo cooperativo se propone el empleo de la rúbrica mostrada en la Tabla 2. En la actualidad no se dispone de cuantificadores para la evaluación del trabajo cooperativo realizado, pero dicha rúbrica se aplicará en años futuros para ver, tanto la eficiencia, como la evolución, de las habilidades referentes al trabajo cooperativo de los estudiantes. La contribución de las tutorías en la nota final de la asignatura es de un $10 \%$. Se propone que un $50 \%$ de dicha nota evalúe el proceso y el otro $50 \%$ evalúe el resultado obtenido. La nota del proceso se obtendrá como la media de la evaluación del profesor y la autoevaluación del propio grupo. Mientras que la nota del resultado obtenido se obtendrá como la media de la evaluación del profesor y la del resto de grupos de trabajo.

\section{Conclusiones}

La técnica de aprendizaje cooperativo basada en el empleo de Puzle de Aronson ha sido utilizada por primer año en las tutorías de la asignatura optativa de Aditivos Alimentarios incluida en el Grado de Ciencia y Tecnología de los Alimentos. Los resultados obtenidos, a pesar de preliminares, han sido muy positivos, no solo respecto al rendimiento académico de los estudiantes, sino también respecto a la mejora de su capacidad para trabajar en grupo. Esta estrategia ha fomentado el desarrollo de las habilidades sociales de los alumnos y su capacidad de integración dentro de grupos, reduciendo la competitividad y rivalidad entre sus miembros. Además ha capacitado a los alumnos para la resolución creativa de problemas, y a la adaptación y aplicación de los conocimientos teóricos a situaciones reales. 
Tabla 2. Rúbrica propuesta para evaluar la capacidad de cooperación del grupo para solucionar un problema propuesto.

\begin{tabular}{|c|c|c|c|c|}
\hline & Excepcional & Admirable & Aceptable & Mejorable \\
\hline $\begin{array}{l}\text { Participación } \\
\text { grupal }\end{array}$ & $\begin{array}{l}\text { Todos los } \\
\text { estudiantes } \\
\text { participan } \\
\text { activamente en la } \\
\text { actividad }\end{array}$ & $\begin{array}{l}\text { La mayoría de los } \\
\text { estudiantes } \\
\text { participan } \\
\text { activamente }\end{array}$ & $\begin{array}{l}\text { Al menos la mitad } \\
\text { de los estudiantes } \\
\text { presentan ideas } \\
\text { propias }\end{array}$ & $\begin{array}{l}\text { No existe } \\
\text { participación activa. }\end{array}$ \\
\hline $\begin{array}{l}\text { Roles dentro del } \\
\text { grupo }\end{array}$ & $\begin{array}{l}\text { Cada estudiante } \\
\text { tiene un rol } \\
\text { asignado bien } \\
\text { definido. }\end{array}$ & $\begin{array}{l}\text { Cada estudiante } \\
\text { tiene un rol } \\
\text { asignado, pero no } \\
\text { está claramente } \\
\text { definido o no es } \\
\text { consistente. }\end{array}$ & $\begin{array}{l}\text { Hay roles asignados } \\
\text { a los estudiantes, } \\
\text { pero no se adhieren } \\
\text { consistentemente a } \\
\text { ellos. }\end{array}$ & $\begin{array}{l}\text { No hay ningún } \\
\text { esfuerzo de asignar } \\
\text { roles a los } \\
\text { miembros. }\end{array}$ \\
\hline $\begin{array}{l}\text { Responsabilidad } \\
\text { compartida }\end{array}$ & $\begin{array}{l}\text { Todos comparten } \\
\text { por igual la } \\
\text { responsabilidad } \\
\text { sobre la tarea }\end{array}$ & $\begin{array}{l}\text { La mayor parte de } \\
\text { los miembros del } \\
\text { grupo comparten } \\
\text { la responsabilidad } \\
\text { en la tarea }\end{array}$ & $\begin{array}{l}\text { La responsabilidad } \\
\text { es compartida por la } \\
\text { mitad de los } \\
\text { integrantes del } \\
\text { grupo }\end{array}$ & $\begin{array}{l}\text { La responsabilidad } \\
\text { recae en una sola } \\
\text { persona }\end{array}$ \\
\hline $\begin{array}{l}\text { Delegación de } \\
\text { responsabilidad }\end{array}$ & $\begin{array}{l}\text { Cada estudiante } \\
\text { en el grupo puede } \\
\text { explicar qué } \\
\text { información es } \\
\text { necesaria para el } \\
\text { grupo y qué } \\
\text { información es } \\
\text { responsable de } \\
\text { localizar. }\end{array}$ & $\begin{array}{l}\text { Cada estudiante } \\
\text { en el grupo puede } \\
\text { explicar qué } \\
\text { información es } \\
\text { responsable de } \\
\text { localizar. }\end{array}$ & $\begin{array}{l}\text { Cada estudiante en } \\
\text { el grupo puede, con } \\
\text { la ayuda de sus } \\
\text { compañeros, } \\
\text { explicar qué } \\
\text { información es } \\
\text { responsable de } \\
\text { localizar. }\end{array}$ & $\begin{array}{l}\text { Uno o más } \\
\text { estudiantes en el } \\
\text { grupo no pueden } \\
\text { explicar qué } \\
\text { información ellos } \\
\text { son responsables de } \\
\text { localizar. }\end{array}$ \\
\hline $\begin{array}{l}\text { Calidad de } \\
\text { interaccion }\end{array}$ & $\begin{array}{l}\text { Todos los } \\
\text { alumnos se } \\
\text { expresan } \\
\text { adecuadamente, } \\
\text { saben escuchar y } \\
\text { se respetan los } \\
\text { puntos de vista y } \\
\text { opiniones de los } \\
\text { demás }\end{array}$ & $\begin{array}{l}\text { La mayor parte de } \\
\text { los alumnos se } \\
\text { expresa } \\
\text { adecuadamente, } \\
\text { sabe escuchar y } \\
\text { respeta los puntos } \\
\text { de vista y } \\
\text { opiniones de los } \\
\text { demás }\end{array}$ & $\begin{array}{l}\text { Una minoria de los } \\
\text { alumnos se expresa } \\
\text { adecuadamente, } \\
\text { sabe escuchar y } \\
\text { respeta los puntos de } \\
\text { vista y opiniones de } \\
\text { los demás }\end{array}$ & $\begin{array}{l}\text { Existe muy poca } \\
\text { interacción, } \\
\text { conversación muy } \\
\text { breve, distracciones } \\
\text { o desinterés. }\end{array}$ \\
\hline $\begin{array}{l}\text { Calidad de las } \\
\text { fuentes }\end{array}$ & $\begin{array}{l}\text { Se han utilizado } \\
\text { los apuntes de la } \\
\text { signatura y se han } \\
\text { ampliado en } \\
\text { búsquedas por } \\
\text { internet y } \\
\text { legislación oficial. }\end{array}$ & $\begin{array}{l}\text { Se han utilizado } \\
\text { los apuntes de la } \\
\text { signatura y se han } \\
\text { ampliado en } \\
\text { búsquedas por } \\
\text { internet. }\end{array}$ & $\begin{array}{l}\text { Se han utilizado los } \\
\text { apuntes de la } \\
\text { asignatura } \\
\text { exclusivamente. }\end{array}$ & $\begin{array}{l}\text { No se ha utilizado } \\
\text { ningún tipo de } \\
\text { fuente. }\end{array}$ \\
\hline $\begin{array}{l}\text { Se han cumplido } \\
\text { los objetivos del } \\
\text { equipo? }\end{array}$ & $\begin{array}{l}\mathrm{Si} \text {, con gran } \\
\text { probailidad de } \\
\text { implantación. }\end{array}$ & $\begin{array}{l}\text { Sí, con poca } \\
\text { posibilidad de } \\
\text { implantación. }\end{array}$ & Parcialmente & No \\
\hline
\end{tabular}

(cc) EY-NC-ND 2017, Universitat Politècnica de València 


\section{Referencias}

GARCÍA, R., TRAVER, J., y CANDELA, I. (2001). Aprendizaje cooperativo. Fundamentos, características y técnicas. Madrid: CCS.

JOHNSON, D. y JOHNSON, R. (1991). Learning together and alone. Cooperative, competitive and individualistic learning. Needham Heights, Allyn and Bacon.

KAGAN, S. (1994). Cooperative Learning. San Clemente, CA: Kagan

TRAVER, J. A., y GARCÍA, R. (2004). La enseñanza-aprendizaje de la actitud de solidaridad en el aula: una propuesta de trabajo centrada en la aplicación de la técnica puzzle de Aronson, en Revista Española de Pedagogía, 229, pp. 419-437. 